\title{
Comparative analyses on epidemiological characteristics of dengue fever in Guangdong and Yunnan, China, 2004-2018
}

Yujuan Yue ( $\nabla$ yueyujuan@icdc.cn )

Chinese Center for Disease Control and Prevention

Qiyong Liu

China CDC

Xiaobo Liu

China CDC

Haixia Wu

China CDC

Research article

Keywords: Comparative analyses, Epidemiological characteristics, Time-series, Spatial, Crowd, Guangdong, Yunnan

Posted Date: December 9th, 2019

DOI: https://doi.org/10.21203/rs.2.18421/v1

License: (9) This work is licensed under a Creative Commons Attribution 4.0 International License.

Read Full License

Version of Record: A version of this preprint was published at BMC Public Health on July 13th, 2021. See the published version at https://doi.org/10.1186/s12889-021-11323-5. 


\section{Abstract}

Dengue fever occurred most severely in Guangdong and Yunnan, accounting for $93.7 \%$ of indigenous cases and $65.9 \%$ of imported cases in mainland China. Epidemiological and spatial-temporal analysis methods were used to compare epidemiological characteristics of dengue cases in Guangdong and Yunnan, China, 2004-2018, including time-series, spatial and crowd features. Much more indigenous cases were widely distributed in Guangdong, while imported cases were more common in Yunnan. 55,970 and 5,938 indigenous cases occurred in 108 counties, Guangdong and 25 counties, Yunnan. 1,146 and 3,050 imported cases occurred in 84 counties, Guangdong and 72 counties, Yunnan. There existed similar seasonal characteristics from July to November for indigenous cases, but there was a longer peak period for imported cases in Guangdong (May to December) than that in Yunnan (July to December). There existed clustering characteristics for dengue fever. $85.1 \%$ of indigenous cases in Yunnan were located in Ruili City and Jinghong City along the southwestern border. Most dengue cases in Guangdong occurred in the Pearl River Delta region, especially with more than $70.0 \%$ in Guangzhou City. $93.9 \%$ of imported cases in Guangdong and Yunna were from 9 countries of Southeast Asia. Thailand, Cambodia and Malaysia were the main imported origins in Guangdong. Myanmar and Laos were the main imported origins in Yunnan. There was a strong male predominance among imported cases and an almost equal gender distribution for indigenous cases. Most dengue cases were from individuals in 21-50 years old, with $57.3 \%$ and $62.8 \%$ for indigenous cases and $83.2 \%$ and $62.6 \%$ for imported cases in Guangdong and Yunnan, respectively. There existed differences in main occupation compositions of dengue cases. However, there were similar major occupations as housework or unemployment, retiree and businessman for indigenous cases, and similar major occupation as businessman for imported cases. Farmers accounted for a larger proportion of dengue cases in Yunnan. The findings of epidemiological characteristics and differences of dengue fever in Guangdong and Yunnan are helpful to formulate targeted, strategic plans and implement effective public health prevention and control measures in China.

\section{Background}

Dengue fever, one of the most prevalent mosquito-borne diseases in humans, is mainly transmitted by Aedes aegyptiand and Aedes albopictus[1]. There are four distinct serotypes for dengue virus, namely DENV 1-4 [2]. It is endemic in more than 100 countries in Southeast Asia, the Americas, Western Pacific, Africa, and Eastern Mediterranean regions [3]. It has evolved from a sporadic disease to a major public health problem as geographical extension, numbers of cases, and disease severity increases[3]. It is estimated that 390 million people had dengue virus infections with 96 million cases annually worldwide [1].

A total of 655,324 cases and 610 deaths were reported in mainland China from 1978 to 2008. A total of 52,749 cases and six deaths were reported from 2009 to 2014 [4]. A dengue fever outbreak occurred in China, 2014, with 47,127 dengue cases according to Chinese National Notifiable Infectious Disease Reporting Information System (CNNDS) [5]. Dengue fever outbreaks have spread from the southern coastal areas as Guangdong (GD) and Hainan to the relatively northern and western areas including 
Fujian, Zhejiang, and Yunnan (YN), with shorter outbreak intervals as compared to those before the 1990s [6]. The affected regions expanded gradually over the 10-year period, from the coastal provinces (Hainan, GD, Fujian, and Zhejiang) of southern China and provinces (Guangxi and YN) adjacent to Southeast Asian countries to the central provinces of China (Henan) [7].

Most of dengue cases, accounting for more than $80 \%$ of the total dengue cases, were reported in GD, YN, Fujian, and Zhejiang, 2005-2015. The overall number of dengue cases reported from GD was largest, and the number of dengue cases in $\mathrm{YN}$ increased in recent years and had the largest number of cases in 2015 [8]. During 2005-2014, the top two provinces were YN (28.8\% of all imported cases) and GD (18.3\% of all imported cases). During 2005-2014, all 53,053 indigenous cases were limited to just seven provinces. $94.3 \%$ of them were reported in GD, and $3.0 \%$ in YN [7]. ${ }^{7}$ Therefore, dengue fever was the most severe in GD and $\mathrm{YN}$, China. It is of great significance for the prevention and control of dengue fever in mainland China to explore the differences of dengue fever in GD and YN. However, Comparative analyses on epidemiological characteristics of dengue fever in GD and YN were rare. Therefore, this study was to compare epidemiological characteristics from indigenous dengue cases and imported dengue cases in GD and YN, including time-series features, spatial distribution features and crowd features.

\section{Methods}

\section{Data collection}

Dengue cases were defined based on clinical diagnosis and laboratory confirmation according to diagnostic criteria and principles of management for dengue (WS 216-2001, before 2008) or diagnostic criteria for dengue (WS 216-2008, after 2008) [9-10].

Dengue fever is a vector-borne notifiable disease in China. Dengue cases are reported to Chinese Center for Disease Control and Prevention (China CDC) through CNNDS. Dengue case report includes sex, age, occupation, national code of current address, date of illness onset, remarks, etc. There are several kinds of occupations, such as farmer, businessman, student, etc. Daily dengue case reports in GD and YN, China from January 1st 2004 to December 13th 2018 were obtained from CNNDS (http://www.chinacdc.cn/). The vector data of Chinese administrative divisions, which were used for geographical mapping, were obtained from CNNDS. The sixth population census in 2010 and gross domestic production (GDP) was obtained from National Bureau of Statistics of China(http://www.stats.gov.cn/).

\section{Data processing}

According to the remarks of dengue case reports, dengue cases were divided into indigenous cases, imported cases and others. Other cases included what we were not sure how to classify. According to the remarks of dengue case reports, indigenous dengue cases occurred from June to December finally. In order to perform spatial analysis, dengue cases were aggregated at the county level according to national 
codes of current addresses, and then were geocoded and matched to the county-level administrative boundaries using ArcGIS version 10.5 [11].

\section{Data analysis}

Time-series analyses and crowd analyses were conducted using IBM SPSS Statistics version 24.0. The Chi-square test was used to compare the overall discrepancies of dengue cases in sex distributions, age group distributions and career distributions in GD and YN, 2004-2018. Spatial distribution analyses for dengue cases were conducted using ArcGIS version 10.5 [11].

\section{Results}

Common information comparison between GD and YN, China was showed in Table 1. The population ratio between GD and $\mathrm{YN}$ is 2.3, but the area ratio is only 0.5. Compared with Yunnan, Guangdong has a slightly smaller number of districts and counties. The ratio of indigenous dengue cases between GD and YN during 2004-2018 reached 9.4, but the ratio of imported dengue cases was only 0.4 . There were 73,761 dengue cases in mainland China during 2004-2018, among which there were $93.7 \%$ of indigenous dengue cases, $65.9 \%$ of imported dengue cases occurred in GD and YN.

Table 1

Common information comparison between GD and YN, China

\begin{tabular}{|llllll|}
\hline & $\begin{array}{l}\text { Population } \\
\text { in } \mathbf{2 0 1 0}\end{array}$ & $\begin{array}{l}\text { Area } \\
\left.\mathbf{( k m}^{\mathbf{2}}\right)\end{array}$ & $\begin{array}{l}\text { Administrative } \\
\text { divisions }\end{array}$ & $\begin{array}{l}\text { Indigenous dengue cases } \\
\text { in 2014-2018 }\end{array}$ & $\begin{array}{l}\text { Imported dengue cases } \\
\text { in 2014-2018 }\end{array}$ \\
\hline GD & $\begin{array}{l}104320 \\
459\end{array}$ & $\begin{array}{l}177 \\
548\end{array}$ & $\begin{array}{l}123 \text { counties } \\
\text { in 21 cities }\end{array}$ & 55970 & 1146 \\
\hline YN & 45966766 & $\begin{array}{l}383 \\
966\end{array}$ & $\begin{array}{l}129 \text { counties } \\
\text { in 16 cities }\end{array}$ & 5938 & 3050 \\
\hline
\end{tabular}

\section{Comparative analyses of indigenous dengue fever in GD and $\mathrm{YN}$}

\section{Time-series comparative analyses of indigenous dengue cases}

There were 55,970 and 5,938 indigenous dengue cases in GD and YN during 2004-2018, respectively. Indigenous dengue fever had been both more and more serious in recent years (Fig. 1(A)). There both existed seasonal characteristics from July to November for indigenous cases and counties (Fig. 1(B)). No indigenous cases occurred in GD in 2005. Indigenous cases occurred in YN only in 2008 and 2013-2018. Indigenous cases in YN only in 2017, 1,954 cases, exceeded those in GD, and indigenous dengue counties in YN only in 2008 exceeded those in GD. A dengue fever outbreak occurred in GD, 2014, and indigenous cases reached 44,795 , accounting for $80.0 \%$ of indigenous cases in GD during 2004-2018. Indigenous 
cases and counties in October, 2014, GD both reached the largest monthly numbers as 22,505 cases and 97.

\section{Spatial comparative analyses of indigenous dengue cases}

Compared with 108 indigenous dengue counties in GD (87.8\% of its total counties), there were 25 indigenous dengue counties in YN (19.4\% of its total counties). Most of indigenous cases in YN were clustered in the southwestern border, and most of indigenous cases in GD were clustered in the southern coast areas. There were 1,239 indigenous cases in Ruili city, Dehong Dai Jingpo Autonomous Prefecture and 3,803 indigenous cases in Jinghong City, Xishuangbanna Dai Autonomous Prefecture, respectively, which accounted for $85.1 \%$ of indigenous cases in YN. Indigenous cases occurred in all the counties along the southern coast of GD, and there were 12 counties with indigenous cases within $1000-12,359$ cases, which included 7 counties in Guangzhou City (with 70.9\% of indigenous cases in GD), 3 counties in Foshan City (6.9\% of indigenous cases in GD) and 2 counties in Chaozhou City and Zhongshan City (Fig. 2). The most indigenous cases, 12,359 cases, occurred in Baiyun County, Guangzhou City.

\section{Crowd comparative analyses of indigenous dengue cases}

Gender composition of the total indigenous dengue cases in GD differed significantly with that in YN during these years (Chi-square $=6.353, P=0.012(<0.05)$, Table 2 ). There were both slightly more female indigenous cases than male indigenous cases.

Table 2

Information comparison of dengue cases from gender in GD and YN, 2004-2018.

\begin{tabular}{|lllll|}
\hline & \multicolumn{2}{l}{ Indigenous cases } & \multicolumn{2}{l|}{ Imported cases } \\
\hline Gender & GD & YN & GD & YN \\
\hline Male & 27760 & 2843 & 765 & 1638 \\
\hline Female & 28210 & 3095 & 381 & 1412 \\
\hline In all & 55970 & 5938 & 1146 & 3050 \\
\hline
\end{tabular}

Age group composition of the total indigenous dengue cases in GD differed significantly with that in YN during these years (Chi-square $=2.202 E 2, P=0.000(<0.05)$, Table 3). Most of indigenous cases were from individuals in 21-50 years old, with $57.3 \%$ in GD and $62.8 \%$ in YN. The largest proportions were both located in individuals in 21-30 years old. 
Table 3

Information comparison of dengue cases from age group in GD and YN, 2004-2018.

\begin{tabular}{|lllll|}
\hline & \multicolumn{2}{l}{ Indigenous cases } & \multicolumn{2}{l|}{ Imported cases } \\
\hline Age group & GD & YN & GD & YN \\
\hline $0-10$ & 2661 & 287 & 8 & 294 \\
\hline $11-20$ & 4675 & 635 & 60 & 444 \\
\hline $21-30$ & 12003 & 1350 & 379 & 842 \\
\hline $31-40$ & 10267 & 1196 & 369 & 600 \\
\hline $41-50$ & 9782 & 1182 & 206 & 467 \\
\hline $51-60$ & 7539 & 710 & 83 & 258 \\
\hline $61-70$ & 5019 & 348 & 34 & 107 \\
\hline $71-$ & 4024 & 230 & 7 & 38 \\
\hline In all & 55970 & 5938 & 1146 & 3050 \\
\hline
\end{tabular}

Career composition of the total indigenous dengue cases in GD differed significantly with that in YN during these years (Chi-square $=2.153 \mathrm{E} 3, \mathrm{P}=0.000(<0.05)$, Table 4$)$. In GD the top four careers for indigenous cases were housework or unemployment, retiree, businessman and worker, with $23.3 \%$, $12.6 \%, 11.3 \%$ and $9.9 \%$, respectively, however, in $\mathrm{YN}$ the top four careers for indigenous cases were businessman, farmer, housework or unemployment and retiree, with $24.0 \%, 13.6 \%, 12.8 \%$ and $9.2 \%$, respectively. 
Table 4

Information comparison of dengue cases from career in GD and $\mathrm{YN}$, 2004-2018.

\begin{tabular}{|lllll|}
\hline Career & \multicolumn{2}{l}{ Indigenous cases } & \multicolumn{2}{l|}{ Imported cases } \\
\hline GD & YN & GD & YN \\
\hline Cadre & 1740 & 278 & 102 & 49 \\
\hline Worker & 5566 & 322 & 193 & 97 \\
\hline Housework or unemployed & 13030 & 760 & 174 & 199 \\
\hline Retiree & 7065 & 546 & 39 & 18 \\
\hline Rural laborer & 1046 & 149 & 16 & 75 \\
\hline Farmer & 2957 & 807 & 31 & 1122 \\
\hline Businessman & 6336 & 1427 & 301 & 712 \\
\hline Student & 4287 & 480 & 58 & 384 \\
\hline Children & 1463 & 140 & 6 & 155 \\
\hline Else & 1652 & 367 & 54 & 76 \\
\hline Unavailable & 10828 & 662 & 172 & 163 \\
\hline In all & 55970 & 5938 & 1146 & 3050 \\
\hline
\end{tabular}

\section{Comparative analyses of imported dengue fever in GD and YN}

\section{Time-series comparative analyses of imported dengue cases}

There were 1,146 and 3,050 imported dengue cases in GD and YN during 2004-2018, respectively. Imported dengue fever had been both more and more severe in recent years (Fig. 3(A)). No imported cases occurred in YN in 2004. There were imported cases in GD all the years around 2014-2018. The imported cases reached a peak of 1,468 cases in YN, 2017, accounting for $48.1 \%$ of its imported cases. The peak periods of imported cases and counties in $\mathrm{YN}$ were both from July to December, however, those in GD were both from May to December (Fig. 3(B)).

\section{Spatial comparative analyses of imported dengue cases}

Compared with 84 imported dengue counties in GD (68.3\% of its total counties), there were 72 imported dengue counties in YN (55.8\% of its total counties). Most of imported cases in YN were clustered in the southwestern border, and most of indigenous cases in GD were clustered in the Pearl River Delta region. The most imported cases at the county level in YN, 1,745 cases, were located in Ruili City, Dehong Dai 
Jingpo Autonomous Prefecture. Imported cases within 50-300 cases at the county level in YN were clustered in the border areas as Xishuangbanna City, Lincang City and Dehong City. However, imported cases at the county level in GD were all below 100 cases. Imported cases within 50-100 cases in GD were concentrated in the coastal areas of Guangzhou City, Dongguan City, Shenzhen City and Zhongshan City. 2,792 imported cases, accounting for $91.5 \%$ of the total imported cases in $\mathrm{YN}$, occurred in the counties with more than 50 cases. And 667 imported cases, accounted for $58.2 \%$ of the total imported cases in GD, occurred in the counties with more than 50 cases(Fig. 4).

Imported cases in GD were from 42 countries, 19 of which imported cases in YN were from. There were 9 imported countries from Southeast Asia, namely Vietnam, Laos, Cambodia, Thailand, Myanmar, Malaysia, Singapore, Indonesia and Philippines, from which there were 3,939 imported cases, accounting for $93.9 \%$ of the total imported cases in GD and YN. And there were 2,854 imported cases from Myanmar, which accounted for $68.0 \%$ of the total imported cases. The imported cases within $100-300$ cases were distributed in Laos, Thailand, Malaysia, Indonesia and Cambodia (Fig. 5). Thailand, Cambodia and Malaysia were the top three sources of imported cases in GD, with 220 cases, 171 cases and 152 cases, respectively. Myanmar, Laos and Thailand were the top three sources of imported cases in YN, with 2,793 cases, 122 cases and 49 cases, respectively.

\section{Crowd comparative analyses of imported dengue cases}

Gender composition of the total imported dengue cases in GD differed significantly with that in YN during these years (Chi-square $=57.962, \mathrm{P}=0.000(<0.05)$, Table 5). There were both more male imported cases than female imported cases.

Table 5

GDP comparison in GD and YN, 2018

\begin{tabular}{|lll|}
\hline & GD & YN \\
\hline GDP (0.1 Billion RMB) & 97277.77 & 17881.12 \\
\hline The primary industry (0.1 Billion RMB) & 3831.44 & 2498.86 \\
\hline The secondary industry (0.1 Billion RMB) & 40695.15 & 6975.44 \\
\hline The third industry (0.1 Billion RMB) & 52751.18 & 8424.82 \\
\hline Per capita GDP (RMB/Person) & 86412 & 37136 \\
\hline
\end{tabular}

Age group composition of the total imported dengue cases in GD differed significantly with that in YN during these years (Chi-square $=2.270 E 2, P=0.000(<0.05)$, Table 6). Most of imported cases were from individuals in the age group of 21-50 years old, with $83.2 \%$ in GD and $62.6 \%$ in YN. The largest proportions were both located in individuals in 21-30 years old.

Career composition of the total imported dengue cases in GD differed significantly with that in $\mathrm{YN}$ during these years (Chi-square $=1.027 \mathrm{E} 3, \mathrm{P}=0.000(<0.05)$, Table 7). In GD the top three careers for imported 
cases were businessman, worker and housework or unemployment, with $26.3 \%, 16.8 \%$ and $15.2 \%$, respectively, however, in YN the top three careers for imported cases were farmer, businessman and student, with $36.8 \%, 23.3 \%$ and $12.6 \%$, respectively.

\section{Discussion}

Dengue fever occurred most severely in GD and $\mathrm{YN}$, accounting for $93.7 \%$ of indigenous cases $(55,970$ cases and 1,146 cases, respectively) and $65.9 \%$ of imported cases (5,938 and 3,050, respectively) in mainland China. Compared with YN, GD had much more indigenous dengue cases but much less imported dengue cases during 2004-2018. However, GD had much more indigenous dengue counties, and also had more imported dengue counties. Dengue fever is closely related with population density and mobility, economic development, traffic development, etc [12-15]. GD has a much smaller area but a great larger population (Table 1). GD also has a much more developed economy than YN (Table 8). Furthermore, $\mathrm{YN}$ is on the border next to Myanmar, where dengue fever was very severe. $68.0 \%$ of imported cases in this study were also from Myanmar. Above all, much more indigenous cases were widely distributed in GD, while imported cases were more common in $\mathrm{YN}$.

There existed similar seasonal characteristics from July to November for indigenous cases, but there was a longer peak period for imported cases in GD (May to December) than that in YN (July to December). Dengue fever is closely related with climate factors such as temperature and rainfall $[8,14-15]$. Yunnan border belongs to the torrid zone, with annual rainfall of $800-1,600 \mathrm{~mm}$. The Southern region of Guangdong belongs to the subtorrid zone, with annual rainfall of more than $1,600 \mathrm{~mm}$. Similar climates led similar seasonal characteristics of indigenous dengue fever there [16]. However, imported dengue fever is more related with economy, population migration, business, travel, etc., and GD had a longer peak period and a much broader imported origins of dengue fever.

Most of dengue cases in GD were located in the Pearl River Delta region, and especially $70.9 \%$ of indigenous cases occurred in 7 counties in Guangzhou City, which is the capital city in GD. $85.1 \%$ of indigenous cases were located in Ruili City and Jinghong City along the southwestern border adjacent to Myanmar, Laos and Vietnam. 93.9\% of the total imported cases in GD and YN were from 9 countries of Southeast Asia, where dengue fever was very severe [17-20]. Thailand, Cambodia and Malaysia were the top three sources of imported cases in GD. Myanmar and Laos were the main sources of imported cases in YN. Dengue outbreaks were triggered by imported cases [21]. Thus both imported cases and indigenous cases were clustered in the similar regions in GD and YN. Therefore, we should focus on the prevention, control and monitoring of the southwestern border of $\mathrm{YN}$ and the Pearl River Delta region of southern GD, especially Ruili City and Jinghong City in YN, as well as Guangzhou City and Foshan City in GD.

By gender, there was a strong male predominance among imported cases and an almost equal gender distribution for indigenous cases. By age group, most of dengue cases were from individuals in 2150 years old, especially $83.2 \%$ of imported cases in GD. This might reflect a population of younger 
working male adults who tend to travel more domestically and regionally and thereby have more exposure risk to dengue [7]. In addition, both indigenous and imported cases across all age group in GD and $\mathrm{YN}$, including the elderly, which is different from other countries in Southeastern Asia where dengue fever is endemic and most dengue cases occur in children or younger adults [22]. The pattern is most likely due to the fact that dengue was not endemic in China and the population in China has very low seroprevalence of dengue antibodies, whereas the population in endemic countries has higher rates of immunity, especially in adults and the elderly [23-25]. By occupation, there were similar major occupations as housework or unemployment, retiree and businessman for indigenous cases, and similar major occupation as businessman for imported cases. Farmers accounted for a larger proportion of dengue cases in YN, which was decided by their industrial structures (Table 8). In order to cope with dengue fever in China, it is necessary to strengthen knowledge propaganda of dengue prevention and control among these occupations.

There also existed some limitation for this research. Data quality of dengue case reports from CNNDS should be improved. Remarks, as a field of case report, describe imported origins as foreign countries or Hong Kong, Macao and Taiwan, China, or simple case definition of imported case or indigenous case, or the process of disease onset and medical treatment, etc. So the description of remarks are not standardized. And a few remarks were missing. However, dengue cases were divided as indigenous or imported cases mainly according to remarks. Therefore there existed a few inevitable errors in case division. There was a large number for unavailable career type. Above all, these factors might influence the result a little in this study.

\section{Conclusion}

Dengue fever occurred most severely in GD and YN. This research provided valuable information on epidemiological characteristics of dengue fever in GD and YN, 2004-2018 by using statistical method and spatial analysis technology. Much more indigenous cases were widely distributed in Guangdong, while imported cases were more common in Yunnan. Dengue fever showed similar seasonal patterns in YN and GD. There existed clustering characteristics for dengue fever. Most dengue cases in Yunnan were clustered in the southwestern border. Most dengue cases in Guangdong occurred in the Pearl River Delta region. Thailand, Cambodia and Malaysia were the top three origins of imported cases in GD. Myanmar and Laos were the main origins of imported cases in YN. There was a strong male predominance among imported cases and an almost equal gender distribution for indigenous cases. Most indigenous and imported cases were both distributed in 21-50 years old. There were similar major occupation origins of dengue cases as Housework or unemployment, Retiree and Businessman. Farmers accounted for a larger proportion of dengue cases in YN. Grasping epidemiological characteristics and differences of dengue fever in GD and $\mathrm{YN}$ is helpful to formulate targeted, strategic plans and implement effective public health prevention and control measures.

\section{Abbreviations}


GD

Guangdong;

YN

Yunnan;

CNNDS

Chinese National Notifiable Infectious Disease Reporting Information System.

\section{Declarations}

\section{Ethics approval and consent to participate}

Not applicable. No human or animal samples were included in the research presented in this article, therefore ethical approval was not necessary for this research according to Measures for ethical review of biomedical research involving human beings (No. 11 of National health commission of the People's Republic of China. http://www.xinyu.gov.cn/fg3tg/201610/f57487a5ef8f4bf3a6df2210956f1963.shtml).

\section{Consent for publication}

Not applicable.

\section{Availability of data and materials}

Daily dengue case reports were obtained from CNNDS (http://www.chinacdc.cn/). The sixth population census in 2010 and gross domestic production (GDP) was obtained from National Bureau of Statistics of China (http://www.stats.gov.cn/).

\section{Competing interest}

The authors declare that they have no competing interests.

\section{Funding}

This study was supported by National Major Science and Technology Project (2017ZX10303404004003 and 2017ZX10303404004004) and National Major Research and Development Program (2016YFC1200802), which can undertake the publication fee.

\section{Author Contributions}

QL initiated the study. YY collected the data, cleaned the data, performed the analysis and drafted the manuscript. XL and HW revised the manuscript. 


\section{Acknowledgements}

We thank the staffs at the hospitals, local health department, and country, district-, prefecture-, and provincial-level CDCs for their valuable assistance in coordinating the data collection.

\section{References}

1. Bhatt S, Gething PW, Brady OJ, Messina JP, Farlow AW, Moyes CL, et al. The global distribution and burden of dengue. Nature 2013;496(7446):504-7.

2. Sang S, Gu S, Bi P, Yang WZ, Xu L, Yang J, et al. Predicting unprecedented dengue outbreak using imported cases and climatic factors in Guangzhou, 2014. PLoS Negl Trop Dis 2015;9(5)e0003808, doi:http://dx.doi.org/10.1371/journal. pntd.0003808.

3. Guzman MG, Harris E. Dengue. Lancet 2015;385:453-65.

4. Chen B, Liu QY. Dengue fever in China. Lancet 2015; 385(9978):1621-2.

5. Yue Y, Sun J, Liu X, Ren D, Liu Q, Xiao X, et al. Spatial Analysis of Dengue Fever and Exploring Its Environmental and Socio-economic Risk Factors Using Ordinary Least Squares: A Case Study in five Districts, Guangzhou City, China, 2014. International Journal of Infectious Disease 2018;75:39-48.

6. Wu J, Lun Z, James A. Review: dengue fever in Mainland China. Am J Trop Med Hyg 2010; 83(3):664-71.

7. Lai SJ, Huang ZJ, Zhou H, Anders KL, Perkins TA, Yin W, et al. The changing epedemiology of dengue in china 1990-2014: a descriptive analysis of 25 years of nationwide surveillance data. BMC Med 2015;13:100.

8. Sun JM, Lu L, Wu HX, Yang J, Xu L, Sang SW, et al. Epidemiological trends of dengue in mainland China, 2005-2015. International Journal of Infectious Disease 2017;57:86-91.

9. Ministry of Health of the People's Republic of China. Diagnostic criteria and principle of management of dengue(WS 216-2001)[in Chinese]. Beijing: Standards Press of China 2001; 1-12.

10. Ministry of Health of the People's Republic of China. Diagnostic criteria for dengue (WS 216-2008) [in Chinese]. Beijing: People's Medical Publishing House 2008; 1-17.

11. ESRI. ArcGIS 10.3 help. ESRI Press 2017.

12. Lai LW. Influence of environmental conditions on asynchronous outbreaks of dengue disease and increasing vector population in Kaohsiung, Taiwan. Int J Environ Health Res 2011;21(2):133-46.

13. Lippi CA, Stewart-Ibarra AM, Muñoz AG, Borbor-Cordova MJ, Mejía R, Rivero K, et al. The social and spatial ecology of dengue presence and burden during an outbreak in Guayaquil, Ecuador, 2012. Int J Environ Res Public Health 2018; 15:827.

14. Liu K, Sun J, Liu X, Li R, Wang Y, Lu L, et al. Spatiotemporal patterns and determinants of dengue at county level in China from 2005-2017. International Journal of Infectious Disease 2018;77:96-104.

15. Liu Q, Xu W, Lu S, Jiang J, Zhou J, Shao Z, et al. Landscape of emerging and re-emerging infectious diseases in China impact of ecology, climate, and behavior. Front. Med. 2018;12(1): 3-22. 
16. 16.Yue YJ, Liu XB, Xu M, Ren DS, Liu QY. Epidemiological dynamics of dengue fever in mainland China, 2014-2018. International Journal of Infectious Disease 2019;86: 82-93.

17. Kurniawati D. Rising number of dengue fever cases in Indonesia. 2013. http://www.aseantoday.com/2013/07/rising-number-of-den-gue-fever-cases-in-indonesia/.

18. Lefevre AS. Thailand suffers worst dengue epidemic in more than 20 years. 2013. http://www.trust.org/item/20131024100249-aqxiv/.

19. Ng LC, Chem YK, Koo C, Mudin RN, Amin FM, Lee KS, et al. 2013 dengue outbreaks in Singapore and Malaysia caused by different viral strains. Am J Trop Med Hyg 2015; 92:1150-5.

20. Kutsuna S, Kato Y, Moi ML, Kotaki A, Ota M, Shinohara K, et al. Autochthonous dengue fever, Tokyo, Japan, 2014. Emerg Infect Dis 2015;21:517-20.

21. Ling F, Fan W, Lin J, Yan J, Lv H, Fu T, et al. Epidemiological survey on a dengue outbreak in Yiwu, Zhejiang Province. Dis Surveillance 2010; 25:757-9.

22. World Health Organization: Dengue fever and dengue hemorrhagic fever. 2014. http://www.who.int/mediacentre/factsheets/fs117/en (2009).Accessed 27 July 2014

23. Xu G, Dong H, Shi N, Liu S, Zhou A, Cheng Z, et al. An outbreak of dengue virus serotype 1 infection in Cixi, Ningbo, People's Republic of China, 2004, associated with a traveler from Thailand and high density of Aedes albopictus. Am J Trop Med Hyg. 2007; 76:1182-8.

24. Huang XY, Ma HX, Wang HF, Du YH, Su J, Li XL, et al. Outbreak of dengue fever in central China, 2013. Biomed Environ Sci. 2014;27:894-7.

25. Guo RN, Lin JY, Li LH, Ke CW, He JF, Zhong HJ, et al. The prevalence and endemic nature of dengue infections in Guangdong, South China: an epidemiological, serological, and etiological study from 2005-2011. PLoS One 2014; 9:e85596.

\section{Figures}


A

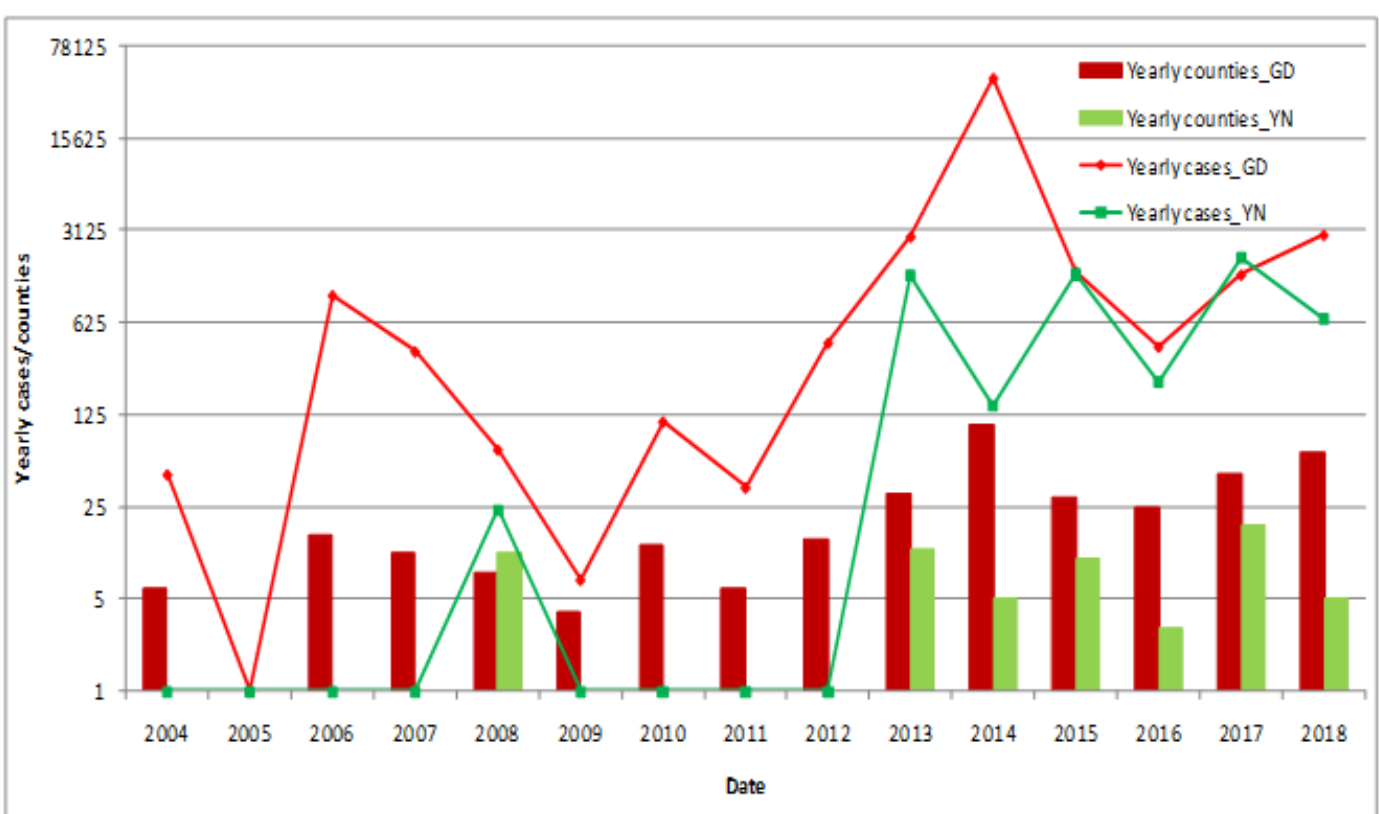

B

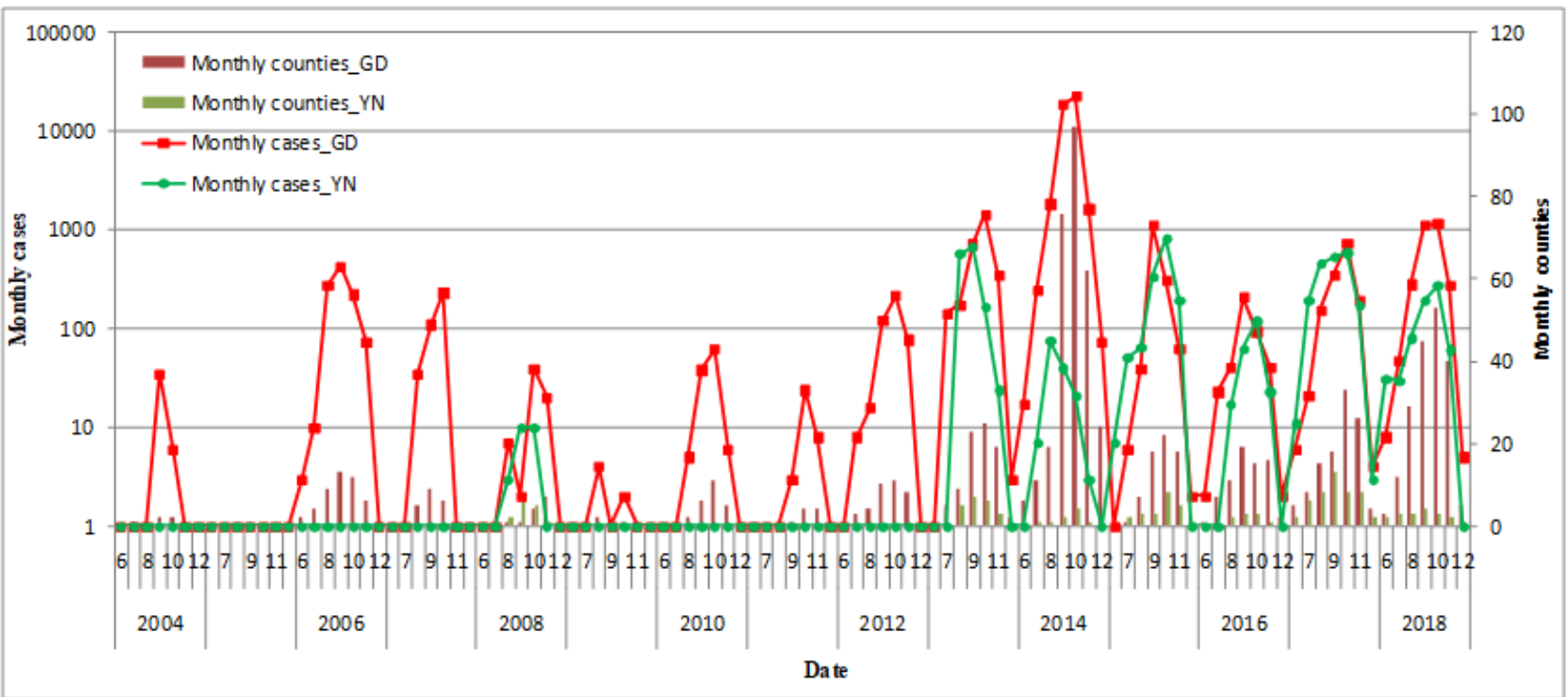

Figure 1

Time-series mapping of indigenous dengue fever in GD and YN, 2004-2018. A. Yearly indigenous fever. B. Monthly indigenous fever. 


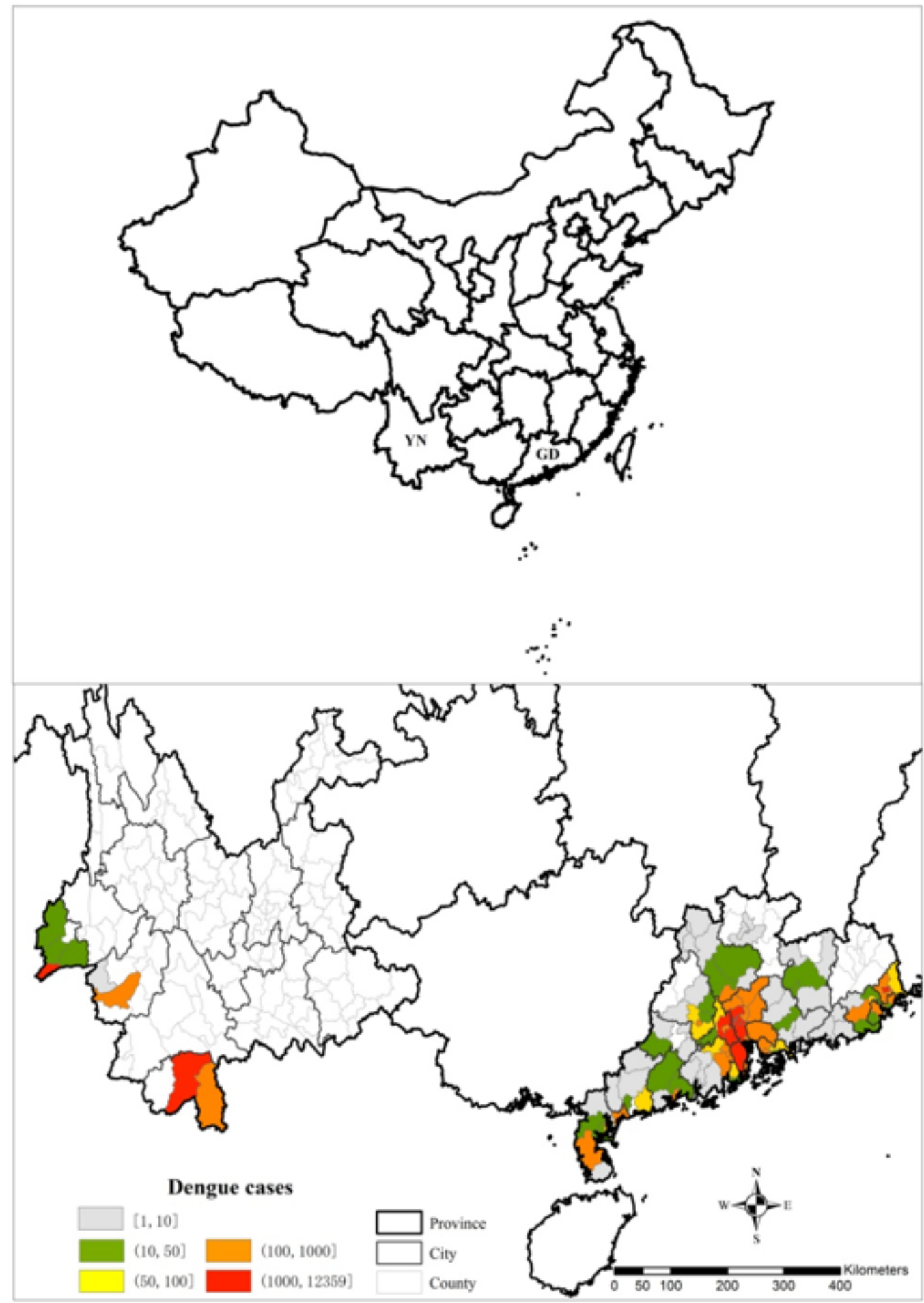

Figure 2

Spatial distribution of indigenous dengue cases in GD and YN, 2004-2018. Note: The designations employed and the presentation of the material on this map do not imply the expression of any opinion whatsoever on the part of Research Square concerning the legal status of any country, territory, city or area or of its authorities, or concerning the delimitation of its frontiers or boundaries. This map has been provided by the authors. 
A

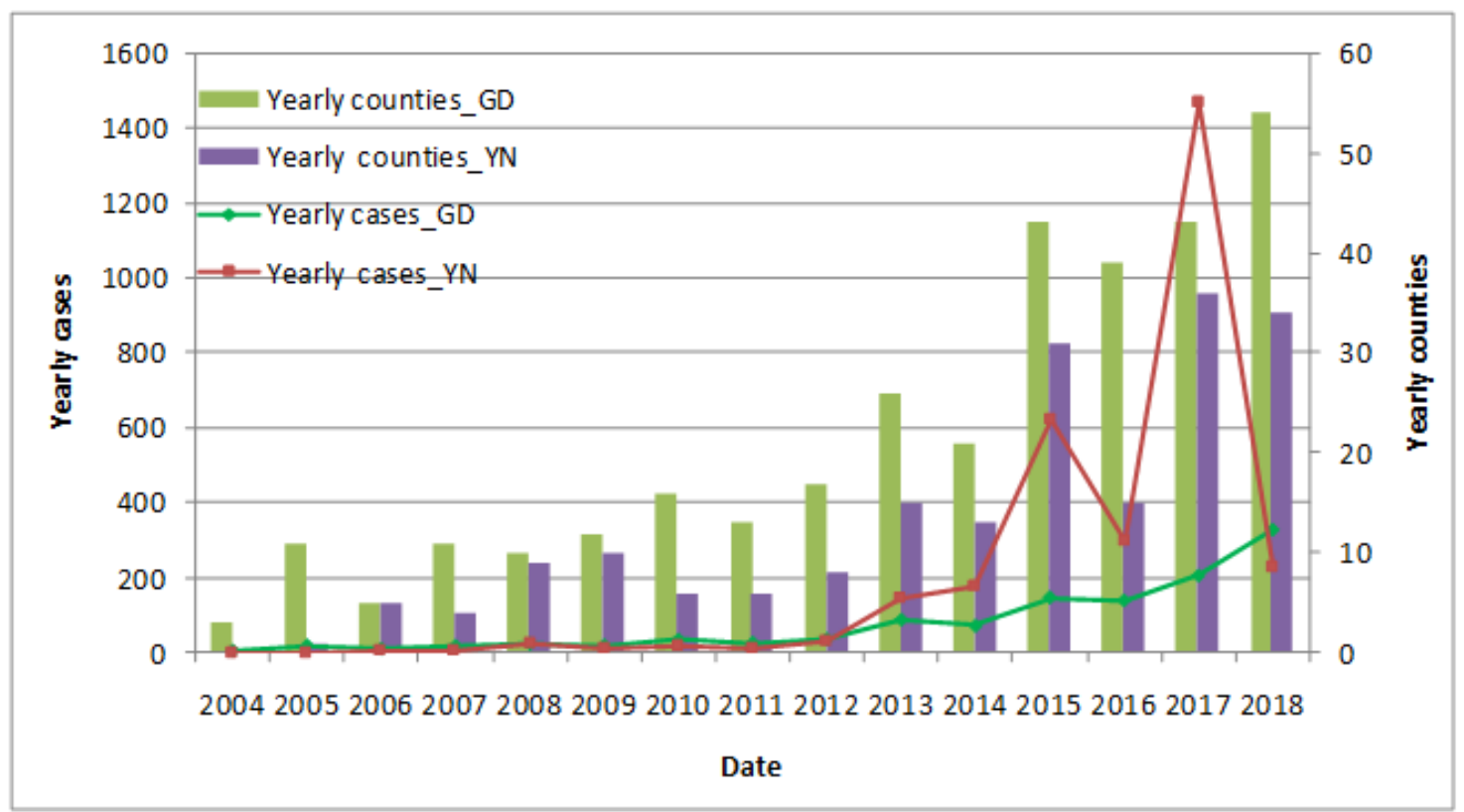

B

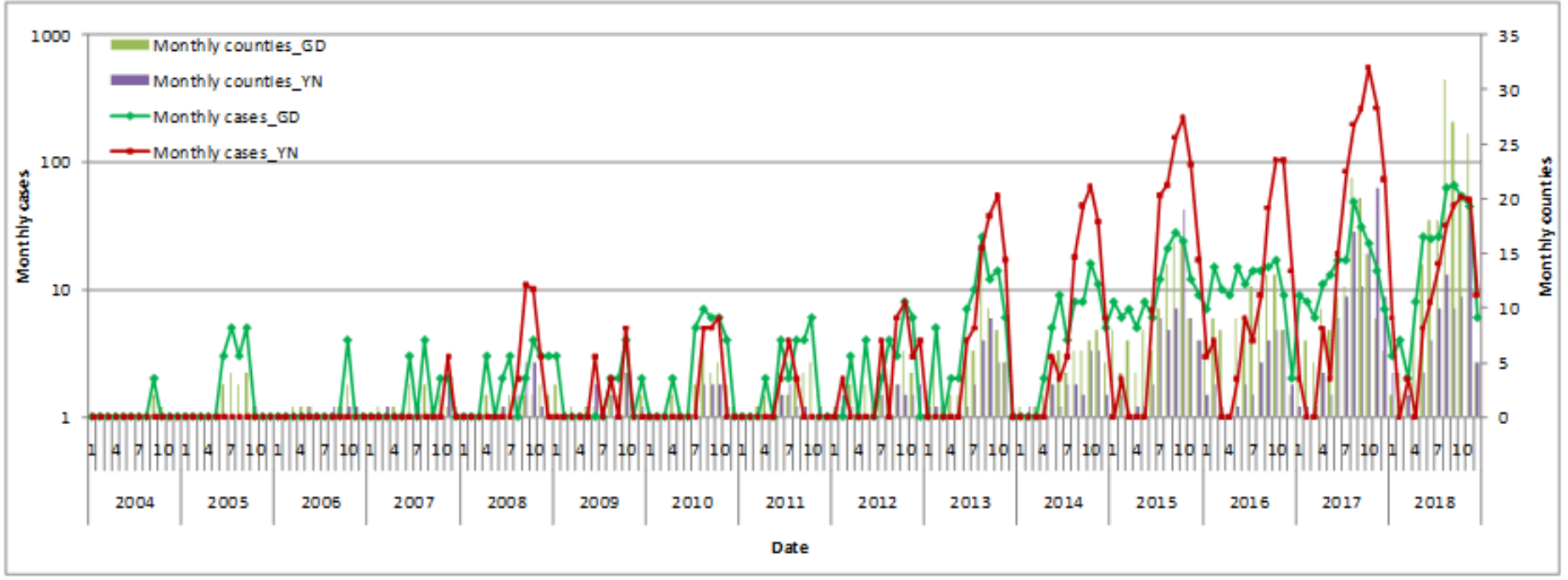

Figure 3

Time-series mapping of imported dengue cases in GD and YN, 2004-2018. A. Yearly imported fever. B. Monthly imported fever. 


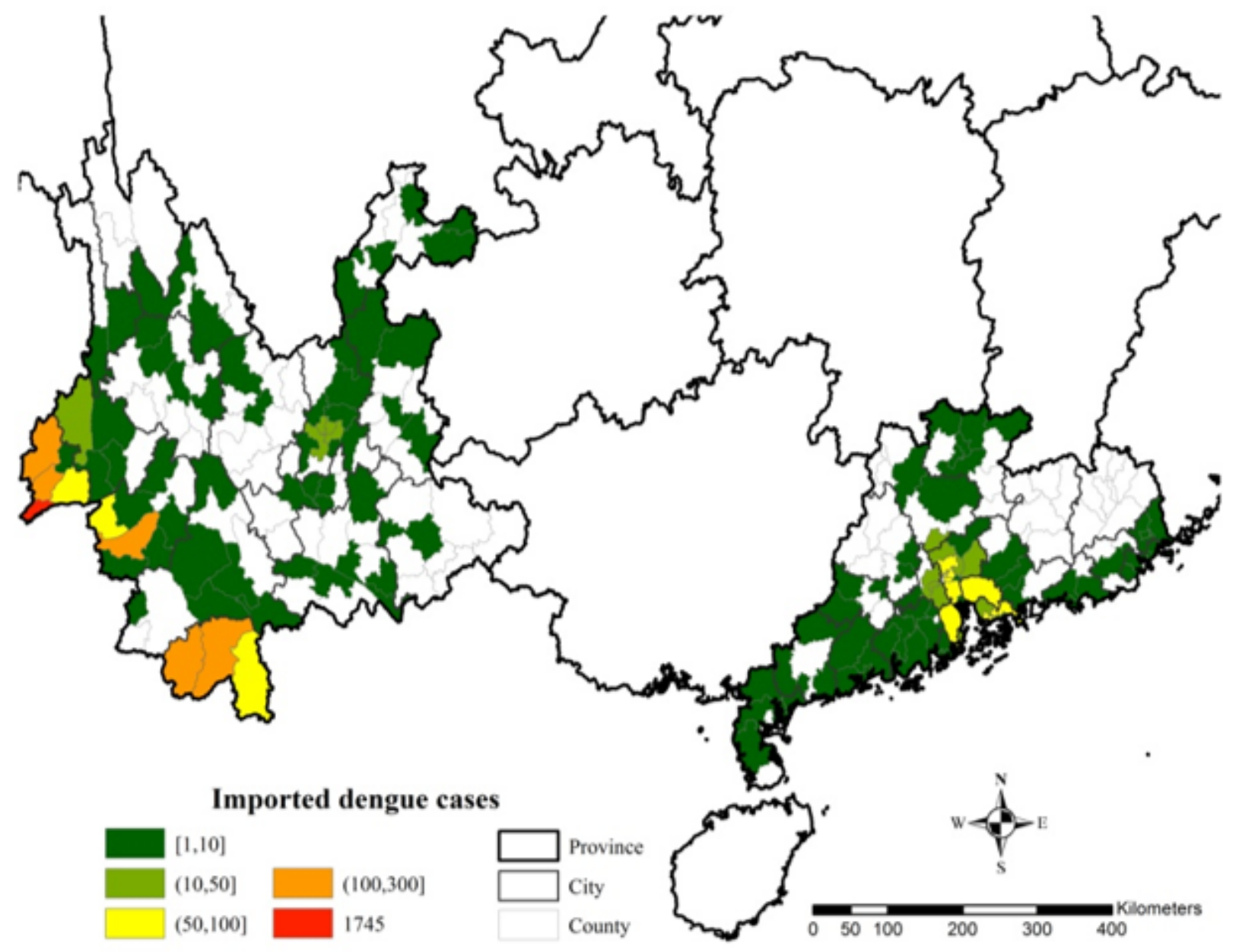

Figure 4

Spatial distribution of imported dengue cases in GD and YN, 2004-2018. Note: The designations employed and the presentation of the material on this map do not imply the expression of any opinion whatsoever on the part of Research Square concerning the legal status of any country, territory, city or area or of its authorities, or concerning the delimitation of its frontiers or boundaries. This map has been provided by the authors. 


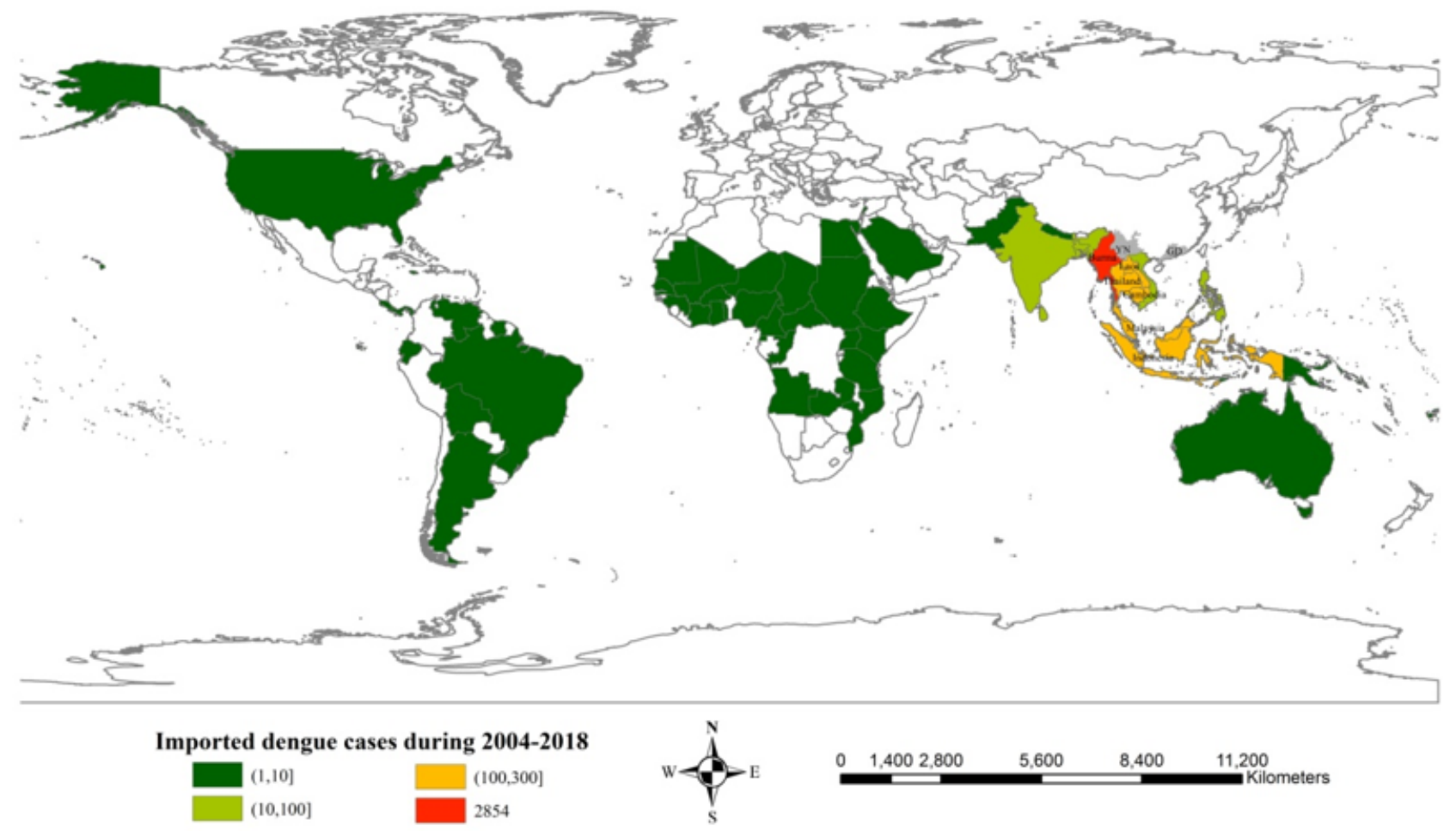

Figure 5

Spatial distribution of imported countries. 